\section{Macros for APA Style}

A set of macros available in three different formatsWordPerfect 5.1 for DOS, WordPerfect 6.0 for Windows, or Microsoft Word 6.0 for Windows or the Macintoshcan be obtained from the authors. The macros enable the writer of a manuscript to easily cite and list references, and report statistical results in the format required by the American Psychological Association (1994).

An initializing macro sets up margins, page headers, and page numbers. A separate document is set up to receive the references. References can be placed in the "References" as they are being cited in the running text, or selected from a previous list of references. The references are automatically formatted and alphabetized, and the citations are placed in the running text. The macros also enable the writer to cite references that already exist in the reference list quickly and easily.

In addition, statistical results can be reported easily. Formats for the most commonly used statistics, includ- ing punctuation, underlining, and alpha levels, are placed in the text; the writer need only insert the appropriate numbers.

A floppy disk ( 3.5 or 5.25 in.) containing the macros and a README file describing how to use the macros can be obtained from the authors. Send $\$ 5.00$ to Marilyn T. Zivian, Department of Psychology, Atkinson College, York University, 4700 Keele Street, North York, ON M3J 1P3, Canada.

\section{REFERENCE}

american Psychological Association. (1994). Publication manual of the American Psychological Association (4th ed.). Washington, DC: Author.

Arthur R. Zivian and Marilyn T. Zivian York University

(Manuscript received June 26, 1996; revision accepted for publication August 19, 1996.) 